\title{
Emodin suppresses silica-induced lung fibrosis by promoting Sirt1 signaling via direct contact
}

\author{
TIAN YANG $^{1}$, JINYUAN WANG ${ }^{2}$, YAMEI PANG $^{1}$, XIAOMIN DANG $^{1}$, \\ HUI REN ${ }^{1}$, YA LIU ${ }^{1}$, MINGWEI CHEN ${ }^{1}$ and DONG SHANG ${ }^{1}$ \\ Departments of ${ }^{1}$ Respiratory and Critical Care Medicine, and ${ }^{2}$ Clinical Laboratory, \\ First Affiliated Hospital of Xi'an Jiaotong University, Xi'an, Shaanxi 710061, P.R. China
}

Received September 2, 2015; Accepted August 26, 2016

DOI: $10.3892 / \mathrm{mmr} .2016 .5838$

\begin{abstract}
Pulmonary silicosis is characterized by lung fibrosis, which leads to impairment of pulmonary function; the specific mechanism remains to be fully elucidated Emodin shows antifibrotic effects in several organs with fibrosis, however, it has not been investigated in pulmonary silicosis. In the present study, the possible mechanism of lung fibrosis and the antifibrotic effect of emodin in silica inhalation-induced lung fibrosis were investigated. Pulmonary silica particle inhalation was used to induce lung fibrosis in mice. Emodin and or the sirtuin 1 (Sirt1) inhibitor, nicotinamide, were used to treat the modeled animals. Pulmonary function was assessed using an occlusion method. The deposition of collagen I and $\alpha$-smooth muscle actin (SMA) in the lung tissue were detected using fluorescence staining; transforming growth factor- $\beta 1$ (TGF- $\beta 1$ ) in the bronchoalveolar lavage fluid (BALF) was examined using an enzyme-linked immunosorbent assay; TGF- $\beta 1 /$ Sirt1/small mothers against decapentaplegic (Smad) signaling activation in lung tissue was also examined. The molecular contacts between emodin were evaluated using liquid chromatography-mass spectrometry analysis. The deposition of collagen I and $\alpha$-SMA in lung tissues were found to be elevated following silica exposure, however, this was relieved by emodin treatment. The pulmonary function of the animals was impaired by silica inhalation, and this was improved by emodin administration. However, the therapeutic effects of emodin on lung fibrosis were impaired by nicotinamide administration. The levels of TGF- $\beta 1$ in the BALF and lung tissue were elevated by silica inhalation, however, they were not affected by either emodin or nicotinamide treatment.
\end{abstract}

Correspondence to: Professor Mingwei Chen or Professor Dong Shang, Department of Respiratory and Critical Care Medicine, First Affiliated Hospital of Xi'an Jiaotong University, 277 West Yanta Road, Xi'an, Shaanxi 710061 P.R. China

E-mail: mingwei_chen36@163.com

E-mail: sd74@vip.sina.com

Key words: lung fibrosis, transforming growth factor- $\beta 1$, small mothers against decapentaplegic, sirtuin 2, emodin
Additionally, emodin was found to increase the expression level of Sirt1, which decreased the level of deacetylated Smad3 to attenuate collagen deposition. Furthermore, the data suggested that there was direct binding between emodin and Sirt1. Sirt1-regulated TGF- $\beta 1 /$ Smad signaling was involved in silica inhalation-induced lung fibrosis. Emodin attenuated this lung fibrosis to improve pulmonary function by targeting Sirt1, which regulated TGF- $\beta 1 / \mathrm{Smad}$ fibrotic signaling.

\section{Introduction}

Individuals with certain occupations, including those in the textile industry, mining and clay manufacturing, are at high risk for the occurrence of pulmonary silicosis (1). As one of the typical pathogens, crystalline silica inhalation can lead to silicosis, which is one of the most frequent forms of occupational lung disease (2). Silicosis is prevalent in developing countries and areas where workers lack appropriate professional protection (3). The pathological characteristics of silicosis are considered to be pulmonary inflammation and fibrosis $(4,5)$. As the fibrosis of silicosis develops, aggravation of the ventilation-perfusion imbalance leads to hypoxemia and respiratory failure (6). Thus, novel therapeutic agents against fibrosis are of clinical significance.

Pulmonary immune cells, particularly alveolar macrophages, are considered to be important in the occurrence and pathogenesis of silicosis (7). When the silica particles are inhaled, macrophages target and enclose the particles by endocytosis. The transcription of inflammatory cytokines, including interleukin-1 $\beta$ (IL-1 $\beta$ ), tumor necrosis factor- $\alpha$ (TNF- $\alpha$ ) and transforming growth factor- $\beta 1$ (TGF- $\beta 1$ ) are initiated (8). Following the binding of TGF- $\beta 1$ and its receptor in lung fibroblasts, the downstream receptor-activated small mothers against decapentaplegic (R-smads), including Smad3, are activated and eventually translocated to the nucleus to initiate the transcription of extracellular matrix (ECM) gene transcription (9).

Emodin, also known as 1,3,8-trihydroxy-6-methylanthraquinone, is one of the agents extracted from several herbaceous plants, including Reynoutria japonica Houtt, and Rheum officinale Baill, which have been used in traditional medicines in Eastern and Southern Asian countries (10). Studies in previous decades have indicated the anti-inflammatory, anticancer and 
antifibrotic effects of emodin (11-13). A previously published study suggested the therapeutic effect of emodin against airway inflammation and fibrosis in an asthma animal model (14).

As a member of a conserved family of $\mathrm{NAD}^{+}$-dependent deacetylases, which are considered to execute multiple fundamental biological functions by deacetylating the lysine residues of several nuclear factors to affect their transcriptional regulatory activities $(15,16)$. It has been suggested that the activation of Sirt1 exerts a negative regulatory effect on TGF- $\beta 1 / \mathrm{Smad} 3$ signaling (15). Evidence has confirmed that Sirt1 can deacetylate Smad3, and subsequently promote the ubiquitination and eventual degradation of R-Smad proteins (16). Therefore, the downstream fibrosis-inducing effect of TGF- $\beta 1$ is inhibited.

Accordingly, the present hypothesized the possible involvement of activation of TGF- $\beta 1 /$ Smad signaling in pulmonary fibrosis in silicosis. Whether Sirt1 was involved in the antifibrotic effects of emodin on silica-induced lung fibrosis was investigated in the present study. It is anticipated that the results from the present study can improve current knowledge of pulmonary fibrosis and provide a theoretical basis for further application of emodin-containing drugs in the treatment of silicosis.

\section{Materials and methods}

Preparation of silica particles and emodin solution. The purity of the silica dust (Sigma-Aldrich; Merck Millipore, Darmstadt, Germany) was used in the present study was $>98 \%$, with a particle diameter of $1-5 \mu \mathrm{m}$. The silica particles were sterilized by being boiled at $120^{\circ} \mathrm{C}$ for $30 \mathrm{~min}$ and then washed with sterilized water and dried. For intratracheal administration, the particles were resuspened in sterile saline and sonicated for $10 \mathrm{~min}$. The emodin (Sigma-Aldrich; Merck Millipore) was dissolved in DMSO and adjusted to $0.1 \%(\mathrm{v} / \mathrm{v})$.

Animals and treatments. Female C57BL/6 mice (SPF class; 6 -week old; $\mathrm{n}=50 ; 10 \mathrm{mice} /$ group) were provided by the Animal Experimental Center of Xi'an Jiaotong University (Xi'an, China). The animals were raised in an environment with an artificial 12/12 h day-night cycle, the temperature controlled at $(25 \pm 1)^{\circ} \mathrm{C}$ and the humidity controlled at $(65 \pm 4) \%$. All animals were maintained with standard food and fresh water. All animal experimental procedures were approved by the Experimental Animal Ethics Committee of Xi'an Jiaotong University.

The mice were anesthetized by intraperitoneal (i.p.) injection of pentobarbital sodium ( $2 \% ; 20 \mathrm{mg} / \mathrm{kg}$ body weight). The neck skin was dissected, following which the trachea was exposed by blunt dissection. A 7-gauge needle was then inserted into the trachea to initiate intratracheal administration. A $50 \mu 1$ silica particle suspension, containing $30 \mathrm{mg}$ silica, was administrated to induce lung fibrosis, then neck skin of the surgical site was sutured and cleaned with ethanol and penicillin. The recovery period was 4 weeks. Control animals received administration of an equal volume of sterile saline. Following model establishment, emodin solution was administrated i.p. once per day ( $20 \mathrm{mg} / \mathrm{kg}$ body weight) for a continuous 7-day period. The Sirt1 inhibitor, nicotinamide (Beyotime Institute of Biotechnology, Nantong, China), which is considered to interrupt the deacetylase activity of Sirt1 (17), was administrated once per day to the animals by i.p. injections (500 mg/kg body weight) for 7 days consecutively following model establishment. For control animals, equal volumes of saline were administrated i.p.

Assessment of pulmonary function. Prior to sacrifice of the animals, the pulmonary function was evaluated according to methods described in a previous study (18). Briefly, the mice were anesthetized by i.p. injection of pentobarbital sodium $(2 \% ; 20 \mathrm{mg} / \mathrm{kg}$ body weight), paralyzed via tail vein injection of pancuronium bromide $(5 \mathrm{mg} / \mathrm{kg}$ body weight) and then subjected to mechanical ventilation (FlexiVent; SCIREQ Scientific Respiratory Equipment, Inc., Montréal, QC, Canada). Respiratory frequency was set as 100/min and tidal volume was set as $0.2 \mathrm{ml}$ with a flow rate of $1 \mathrm{ml} / \mathrm{sec}$. The transpulmonary pressure was measured using pressure transducers. Airway resistance, dynamic compliance and elastance were calculated according to the occlusion method described previously (18). Subsequent to establishment of pulmonary function, the mice were sacrificed by overdose of anesthesia with pentobarbital sodium.

Fluorescent staining of $\alpha$-SMA and collagen I in lung tissues. The lung tissue was harvested, trimmed and embedded in OCT compound (Sakura Finetek USA, Inc., Torrance, CA, USA) at $-20^{\circ} \mathrm{C}$ for $20 \mathrm{~min}$. The lung tissue was then cut into slices at a thickness of $5 \mu \mathrm{m}$. The slices were incubated with antibodies against $\alpha$-SMA (ab5694; Abcam, Cambridge, MA, USA) and collagen I (ab34710; Abcam). Following incubation with secondary antibodies conjugated with Alexa 594 (A-11072; Invitrogen; Thermo Fisher Scientific, Inc., Waltham, MA, USA) and Alexa 488 (A27034; Invitrogen; Thermo Fisher Scientific, Inc.), respectively, fluorescent images were observed under a fluorescent microscope (Nikon, Tokyo, Japan).

Determination of bronchoalveolar lavage fluid (BALF) $T G F-\beta 1$ levels. Following sacrifice of the mice, BALF was obtained with the assistance of tracheal cannulation. The BALF was centrifuged at $200 \times g$ for $10 \mathrm{~min}$ at $4^{\circ} \mathrm{C}$, following which the supernatant was collected. The level of TGF- $\beta 1$ was determined using an using enzyme-linked immunosorbent assay kit (R\&D Systems, Inc., Minneapolis, MN, USA) according to the manufacturer's protocol.

Resin preparation. CNBr-activated Sepharose ${ }^{\mathrm{TM}}$ 4B (GE Healthcare, Piscataway, NJ, USA) was used following preparation with $\mathrm{HCl}(1 \mathrm{mmol} / \mathrm{l})$ and washing in coupling buffer $\left(0.1 \mathrm{mmol} / 1 \mathrm{HaHCO}_{3}\right.$ and $\left.0.5 \mathrm{mmol} / \mathrm{l} \mathrm{NaCl}\right)$. For the emodin- loaded affinity column, emodin was dissolved in DMSO $(5 \mathrm{mmol} / \mathrm{l})$ and then mixed into the resin at a ratio of 1:10 emodin:resin. The resin was then rotated for $4 \mathrm{~h}$ at room temperature and washed with deionized water. Any possible remaining active groups were inhibited using capping solution ( $1 \mathrm{mmol} / 1$ ethanolamine) for $2 \mathrm{~h}$ at room temperature. Sirt1 protein $(15 \mu \mathrm{l}$ at a concentration of $10 \mathrm{mg} / \mathrm{ml})$ was added into the resin and then incubated at $4^{\circ} \mathrm{C}$ for $8 \mathrm{~h}$. Following incubation, the excess protein was washed, and the resin was added to loading buffer (Beyotime Insitute of Biotechnology) and boiled. The mixture was then subjected to SDS-PAGE and assessed using western blot analysis. For 

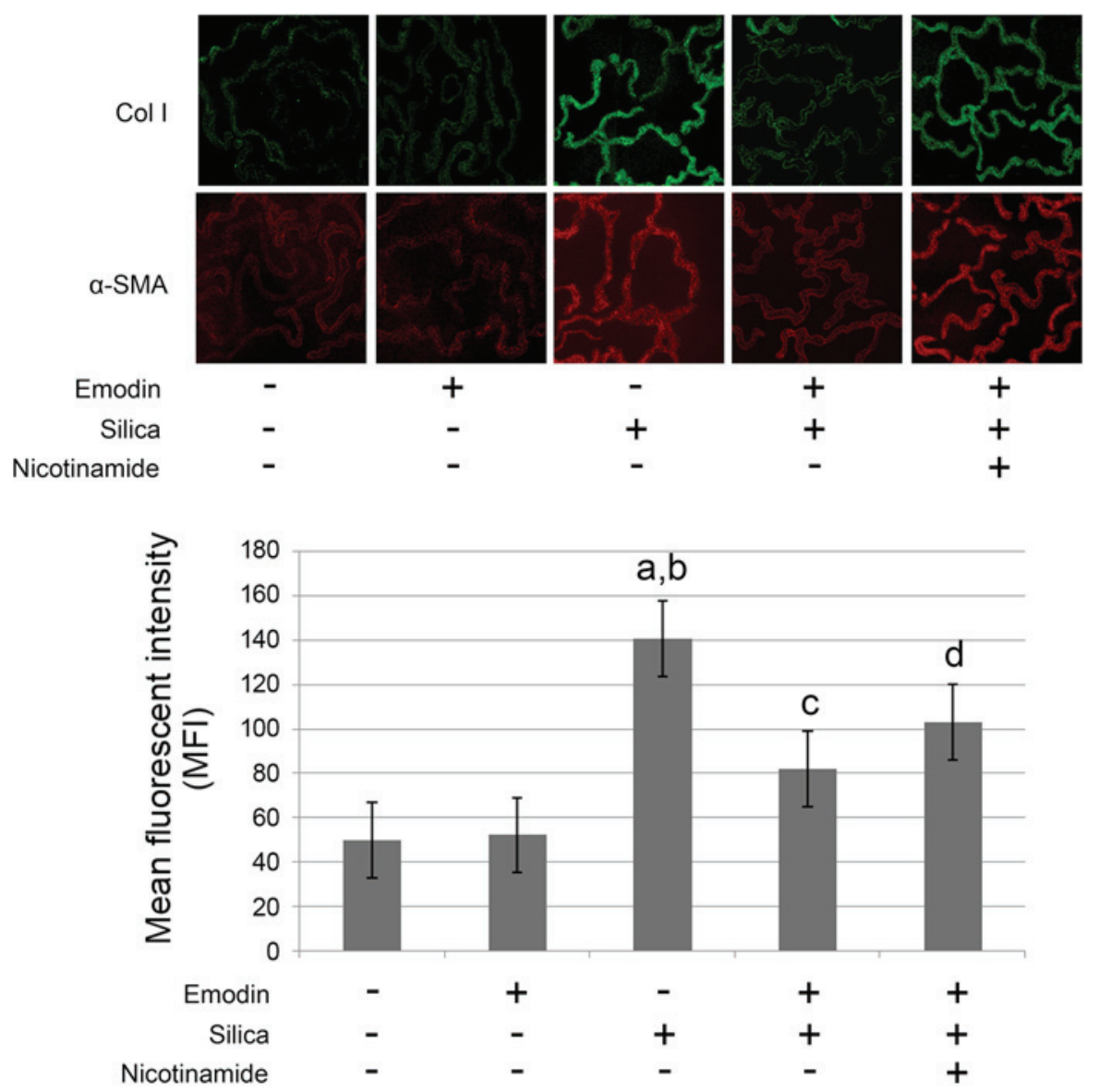

Figure 1. Images captured of the fluorescent staining of Col I and $\alpha$-SMA in lungs harvested from mice with silica inhalation-induced lung fibrosis treated with emodin and/or nicotinamide. The graph below shows the intensity of Col I and $\alpha$-SMA fluorescent staining, presented as the mean \pm standard deviation. Magnification, $\mathrm{x} 200$. ${ }^{\mathrm{a}} \mathrm{P}<0.05$, compared with the untreated control; ${ }^{\mathrm{b}} \mathrm{P}<0.05$, compared with the emodin-treated group; ${ }^{\mathrm{c}} \mathrm{P}<0.05$, compared with the silica-exposed group; ${ }^{\mathrm{d}} \mathrm{P}<0.05$, compared with the emodin-treated silica-exposed group. Col I, collagen I; $\alpha$-SMA, $\alpha$-smooth muscle actin.

the Sirt1-loaded affinity column, Sirt1 protein $(10 \mathrm{mg} / \mathrm{ml}$ Sigma-Aldrich; Merck Millipore) was added to the resin and then rotated at $4^{\circ} \mathrm{C}$ for $8 \mathrm{~h}$, followed by washing with capping solution at room temperature for $2 \mathrm{~h}$. The resin was then treated with emodin solution for $4 \mathrm{~h}$ at room temperature. Following washing with high $\mathrm{pH}$ buffer $(0.1 \mathrm{mmol} / 1$ Tris- $\mathrm{HCl}$ and $0.5 \mathrm{mmol} / 1 \mathrm{NaCl} ; \mathrm{pH} 8)$ and low $\mathrm{pH}$ buffer $(0.1 \mathrm{mmol} / \mathrm{l}$ $\mathrm{AcOH}$ and $0.5 \mathrm{mmol} / 1 \mathrm{NaCl} ; \mathrm{pH}=4)$ three times, a Vivapure C18 spin column (Sartorius AG, Göttingen, Germany) was used to accomplish the desalt process prior to liquid chromatography-mass spectrometry (LC/MS) analysis.

LC/MS analysis. A BioBasic Picofrit C18 capillary column (New Objective, Inc., Woburn, MA, USA) was used to separate the peptides. Using an acetonitrile gradient (0-100\%) with a flow rate of $1 \mathrm{ml} / \mathrm{min}$ for $1 \mathrm{~h}$, the elution was prepared. The 6410B Triple Quadrupole LC/MS (Agilent Technologies, Inc., Santa Clara, CA, USA) system was used for LC/MS in the present study according to the manufacturer's protocol.

Western blot analysis. Activation of the TGF- $\beta 1 / \mathrm{Smad}$ signaling pathway was assessed in the present study using western blot analysis. The pre-cleaned lung tissue was homogenized in RIPA buffer with PMSF. Following centrifugation at $14,000 \times \mathrm{g}$ at $4^{\circ} \mathrm{C}$, the supernatants were used for western blot analysis. The protein concentration was determined using a BCA protein assay kit (Santa Cruz Biotechnology, Inc., Dallas, TX, USA). The proteins $(50 \mu \mathrm{g})$ were then separated by SDS PAGE (4\% stacking gel and $10 \%$ separation gel) vertically and semi-dry transferred onto polyvinylidene fluoride membranes. Antibodies against TGF- $\beta 1$ (ab64715; Abcam), Smad3 (A27034; Cell Signaling Technology, Inc., Danvers, MA, USA), acetylated (Ac)-Smad3 (\#9513; Cell Signaling Technology, Inc,), Sirt1 (\#8469; Cell Signaling Technology, Inc.), $\alpha$-SMA (Abcam), collagen I (Abcam) and GAPDH (\#MA1-16757; Invitrogen; Thermo Fisher Scientific, Inc.) were used to incubate the membranes. Rabbit anti-mouse (sc-358943), bovine anti-goat (sc-2384) and bovine anti-rabbit (sc-2385) secondary antibodies conjugated to horseradish peroxidase (Santa Cruz Biotechnology, Inc.) were used to incubate the membranes. Subsequently, Super Signal West Pico chemiluminescence reagent (Thermo Fisher Scientific, Inc.) was used to develop the membranes and the immunoblots were visualized on $\mathrm{X}$-ray films.

Statistical analysis. The results in the present study are presented as the mean \pm standard deviation and were analyzed using SPSS software (version 16.0; SPSS, Inc., Chicago, IL, USA). Differences between values were evaluated using 
A
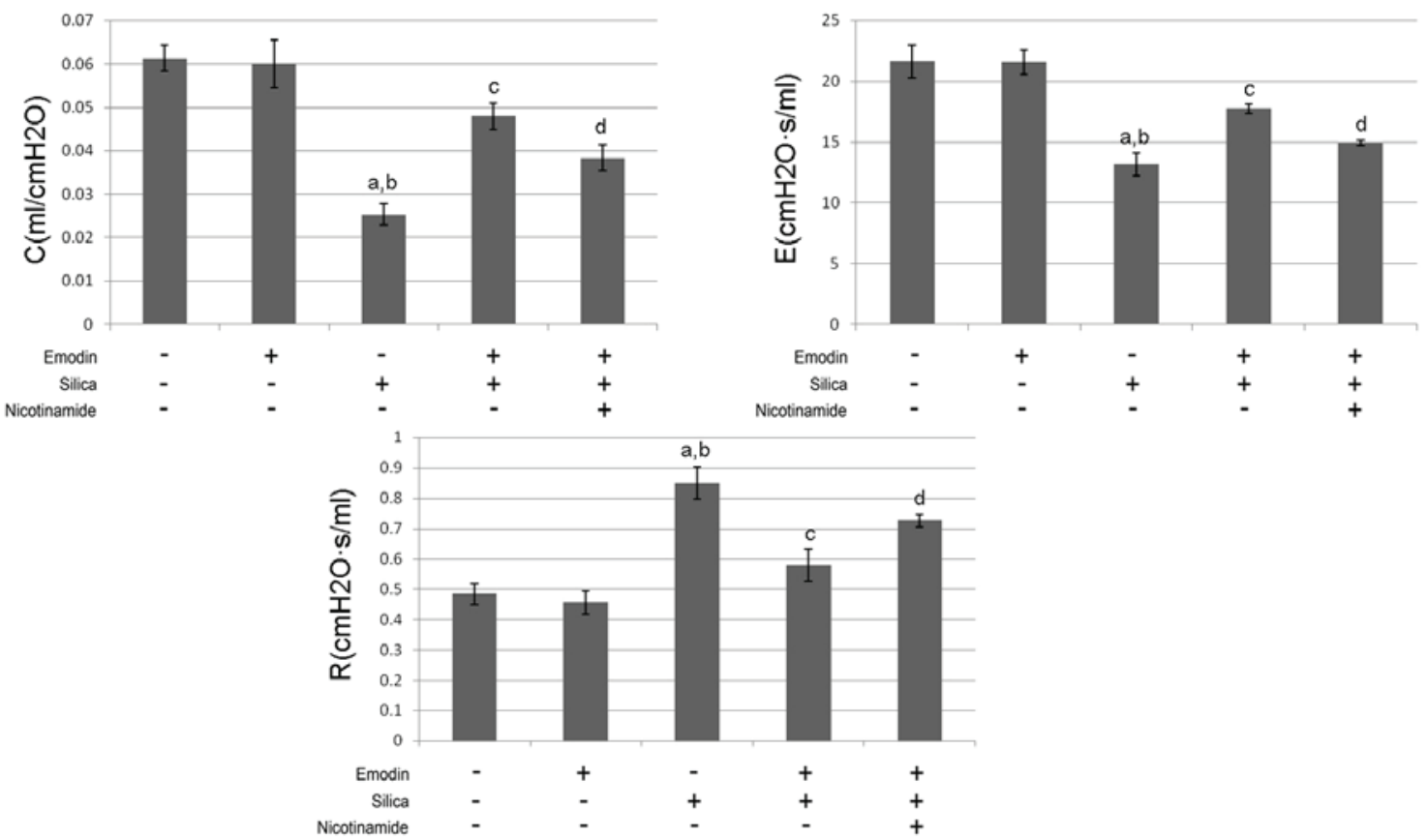

B
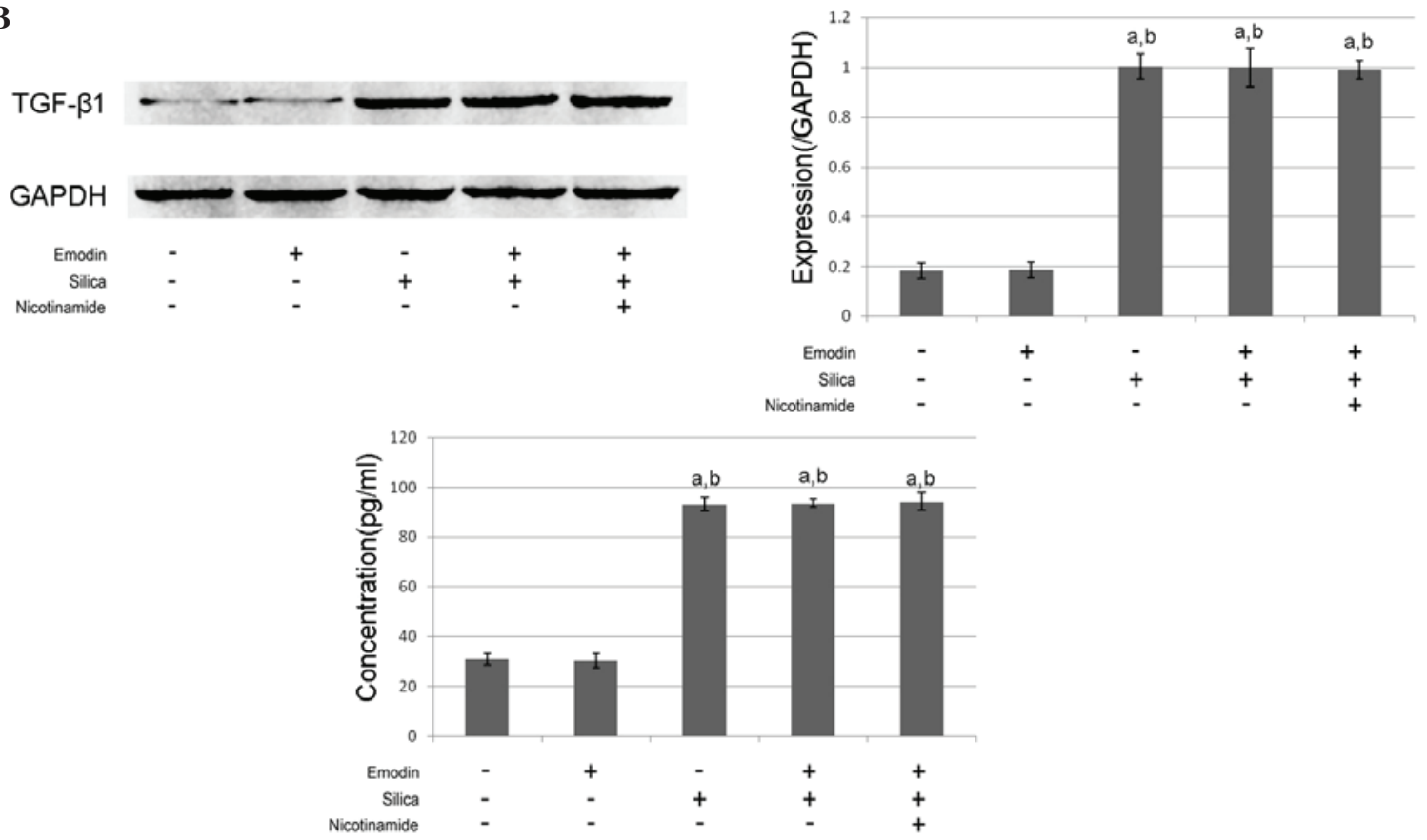

Figure 2. (A) Measurements of respiratory function (C, E and R) were determined using the occlusion method and are presented as the mean \pm standard deviation. (B) Immunoblots of TGF- $\beta 1$ and GAPDH in lung tissues from mice with silica inhalation-induced lung fibrosis treated with emodin and/or nicotinamide. The graph on the left shows the relative expression of TGF- $\beta 1$ (GAPDH was introduced as an internal reference). The graph on the right show the concentrations of TGF- $\beta 1$ in the BALF, determined using an enzyme-linked immunosorbent assay. ${ }^{a} \mathrm{P}<0.05$, compared with the untreated control; ${ }^{\text {b }} \mathrm{P}<0.05$, compared with the emodin-treated group; ${ }^{\mathrm{c}} \mathrm{P}<0.05$, compared with the silica exposed group; ${ }^{\mathrm{d}} \mathrm{P}<0.05$, compared with the emodin-treated silica-exposed group. TGF- $\beta 1$ transforming growth factor- $\beta 1$; $\mathrm{R}$, airway resistance; $\mathrm{C}$, dynamic compliance; E, elastance.

one-way analysis of variance or Student's- $t$-test. $\mathrm{P}<0.05$ was considered to indicate a statistically significant difference.

\section{Results}

Silica exposure induces lung fibrosis and impairs pulmonary functions. As shown in Figs. 1 and 2, compared with the control animals, marked lung fibrosis was observed following exposure of the lungs to silica. The lung fibrosis was evidenced by increased $\alpha$-SMA and collagen I deposition in the lung tissues
(Fig. 1) Furthermore, the pulmonary function was impaired following the induction of lung fibrosis. The airway resistance was increased, whereas the dynamic compliance and elastance were reduced (Fig. 2A). The levels of TGF- $\beta 1$ in the BALF and the lung tissues were markedly increased, compared with the control group (Fig.2B).

Emodin inhibits silica-induced lung fibrosis and improves pulmonary functions without affecting levels of TGF- $\beta 1$ in the BALF and lung tissue. As shown in Figs. 1 and 2, i.p. treatment 


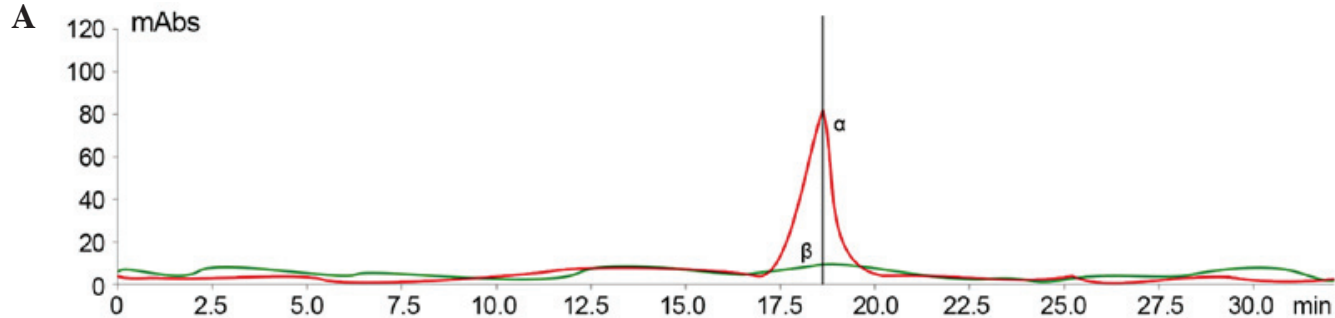

B

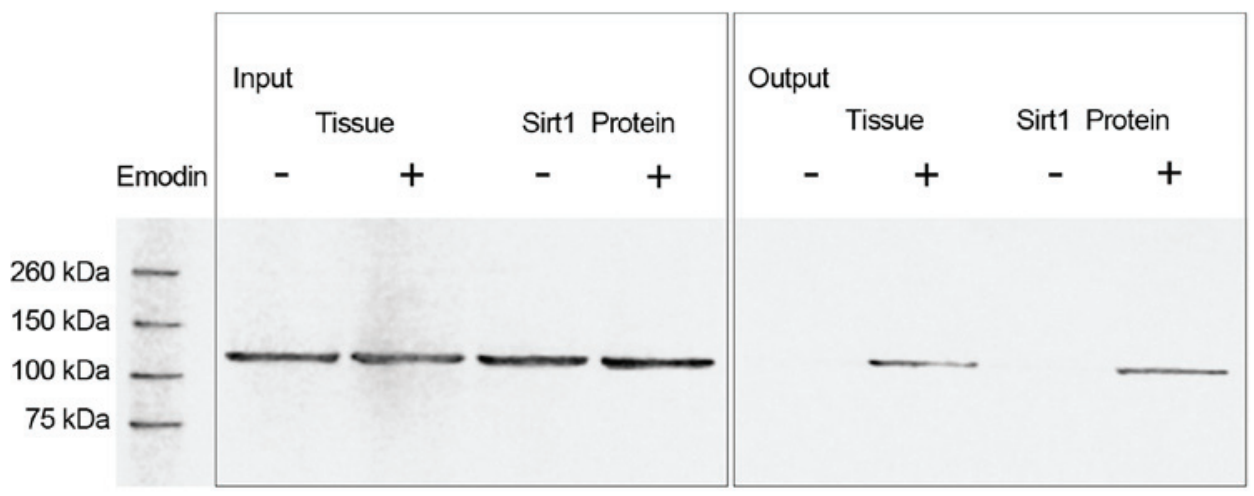

Figure 3. (A) Liquid chromatography-mass spectrometry detection of emodin eluted from Sirtl-loaded affinity columns. The $\alpha$ peak (in red curve) and $\beta$ peak (in green curve) indicate the detected samples from the control and emodin-bound Sirtl-loaded columns, respectively. The $\alpha$ peak represents the specific peak for emodin. (B) Immunoblots of Sirt1 further confirmed the direct binding of emodin to Sirt1. '-' indicates non-emodin-loaded affinity columns, '+' indicates emodin-loaded affinity columns. The samples comprised lung tissue lysate and Sirt1 protein. mAbs, monoclonal antibodies; Sirt1, surtuin 1.
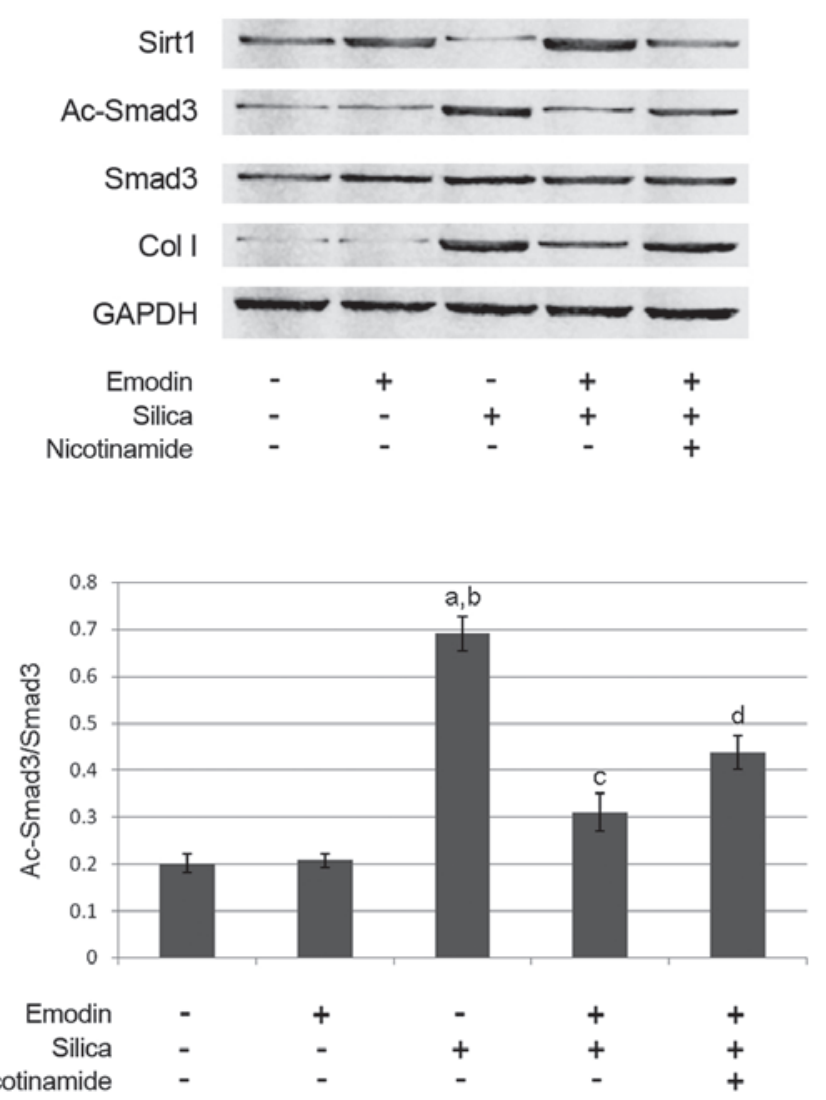
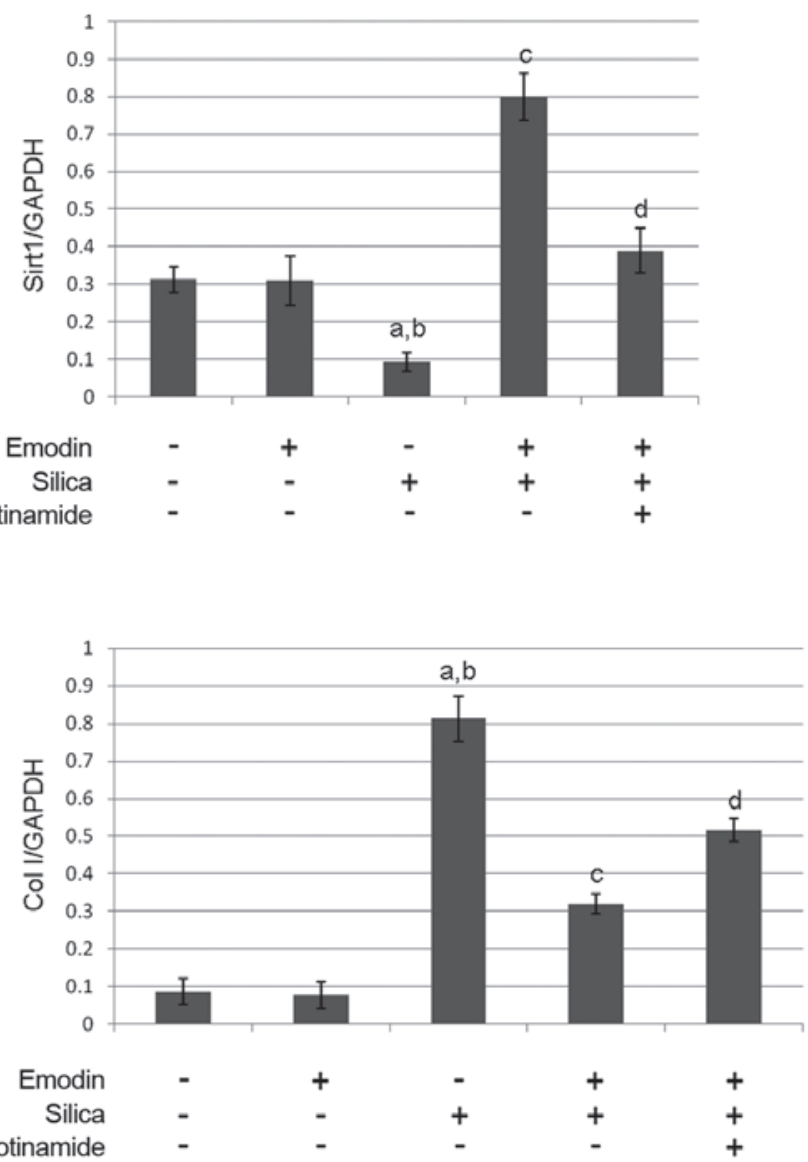

Figure 4. Immunoblots of Sirt1, Ac-Smad3, Smad3, Col I and GAPDH in harvested lungs from mice with silica inhalation-induced lung fibrosis treated with emodin and/or nicotinamide. Graphs show the relative expression levels of Sirt1, Ac-Smad3 and Col I. ${ }^{\mathrm{a} P}<0.05$, compared with untreated control; ${ }^{\mathrm{b}} \mathrm{P}<0.05$, compared with emodin-treated group; ${ }^{\mathrm{C}} \mathrm{P}<0.05$, compared with the silica-exposed group; ${ }^{\mathrm{d}} \mathrm{P}<0.05$, compared with the emodin-treated silica-exposed group. Sirt1, surtuin 1; Ac-Smad3, acetylated small mothers against decapentaplegic 3; Col, collagen I. 
with emodin significantly inhibited lung fibrosis and attenuated the pulmonary functions of the animal models. Furthermore, the administration of emodin did not affect the levels of TGF- $\beta 1$ in the BALF or lung tissue.

Nicotinamide treatment impairs the effects of emodin on lung fibrosis and pulmonary function without affecting levels of $T G F-\beta 1$. The results of the effects of nicotinamide are also shown in Figs. 1 and 2. The mice received i.p. treatment with emodin and nicotinamide following silica inhalation. Compared with the silica-inhaled mice treated with emodin, nicotinamde administration significantly impaired the effects of emodin on inhibiting lung fibrosis and improving pulmonary functions. However, neither emodin nor nicotinamide affected the levels of TGF- $\beta 1$ in the BALF or lung tissue.

Emodin interacts with Sirtl by direct contact. As shown in Fig. 3, LC/MS analysis confirmed that emodin was able to bind to Sirt1. Also shown in Fig. 3, following incubation of the emodin-affinity columns with Sirt1 protein, the results of the western blot analysis suggested that Sirt1 was in contact with emodin.

Emodin elevates the acetylation of Smad3 by increasing the expression of Sirt1. The immunoblots of Sirt1, Smad3, Ac-Smad3, collagen I and GAPDH are shown in Fig. 4. Compared with the control animals, silica inhalation markedly increased Ac-Smad3/Smad3, which subsequently elevated the level of collagen I in the lung tissues of the model animals. Emodin administration increased the expression of Sirt1 and decreased Ac-Smad3/Smad3. However, treatment with the Sirt1 inhibitor, nicotinamide, impaired the effects of emodin on decreasing Ac-Smad3/Smad3 and elevating Sirt1. As a result, nicotinamide undermined the effect of emodin on decreasing the level of collagen I.

\section{Discussion}

In the present study, an animal model of pulmonary silicosis was induced by intratracheal administration of silica particles. Lung fibrosis was clearly identified in these animals. As a result, pulmonary functions were impaired in the mice with silica-induced lung fibrosis, and the present study provided further insight into the possible mechanism underlying silica-induced lung fibrosis. It was found that the TGF- $\beta 1 / \mathrm{Smad} 3$ signaling pathway was activated in silica-induced lung fibrosis. The present study also examined the antifibrotic activity of emodin in silica-induced lung fibrosis. Investigation of the mechanism revealed that, without affecting the expression level of TGF- $\beta 1$ in the lungs, emodin downregulated Smad-induced lung fibrosis by promoting Sirtl signaling via direct binding, which caused the deacetylation of R-Smads to inhibit ECM synthesis and deposition.

Characterized by impaired pulmonary function and hypoxemia, silicosis is considered to be a common occupational disease (19). The chronic inhalation of silicon dioxide particles, which is also referred as silica, is suggested to be the pathogenic factor of lung silicosis. Pathologically, chronic lung inflammation and fibrosis are accepted as the typical characteristics of pulmonary silicosis (20), and the impairment of pulmonary function is initiated and expedited by lung fibrosis (21). In the present study, lung fibrosis was identified following exposure of the lungs to silica particles. Characterized by increased airway resistance, reduced dynamic compliance and elastance, the pulmonary function was impaired, accompanied by the lung fibrosis.

During fibrosis, immune cells are activated and several types of inflammatory cytokines, including interferon- $\gamma$, tumor necrosis factor- $\alpha$, ILs and TGF- $\beta$ are released (22). It is generally accepted that TGF- $\beta$ is one of the most potent initiators of fibrosis by inducing fibroblasts to synthesize ECM, including collagen, laminin and fibronectin (23). On binding to its receptors, the formed complex recruits and triggers the phosphorylation of R-Smads, which further target gene-encoding ECM proteins as transcription factors (24). In the present study, the expression levels of TGF- $\beta 1$ were found to be elevated in the BALF and lung tissue. This result indicated that silica exposure promoted the synthesis and release of TGF- $\beta 1$ from immune cells accumulated in the airway and lung tissues. Previously, it was documented that Smad3 is one of the underlying mechanisms promoting fibrogenesis in response to multiple fibrogenic initiators, including angiotensin II, advanced glycation end products and TGF- $\beta(25,26)$. With the exception of the phosphorylation of R-Smads, accumulating evidence suggests that the acetylation of $\mathrm{R}$-Smads is also a critical signal, which induces the production and deposition of ECM, and can be induced by TGF- $\beta 1$ (15). In the present study, high levels of R-Smad acetylation were found, which led to lung fibrosis, characterized by the deposition of $\mathrm{ECM}$ in the lung tissue.

Natural agents of herbal origin have attracted substantial attention in previous decades due to their multiple biological activities. A number of these agents, including curcumin, emodin and matrine, have been shown to exert antifibrotic activity $(27,28)$. Thus, these agents are of potential therapeutic value in the treatment of fibrosis of pulmonary silicosis. In the present study, the administration of emodin resulted in lung fibrosis being relieved, and the pulmonary function was improved in the animals with lung fibrosis. Further investigation showed that there was molecular binding between emodin and Sirt1. These results indicated that emodin may have a regulatory effect on Sirt1.

In the present study, it was found that, in mice with silica-induced lung fibrosis, emodin treatment not only increased the expression level of Sirtl in lung tissues, but also significantly promoted the deacetylation of R-Smads in the lung tissue without affecting the levels of TGF- $\beta 1$ in the lung tissue or BALF. Furthermore, treatment with the Sirt1 inhibitor, nicotinamide, suppressed the emodin-induced deacetylation of R-Smads. In conclusion, emodin showed significant antifibrotic activity in inhibiting lung fibrosis in pulmonary silicosis, improving pulmonary function. Mechanically, the present study provided evidence confirming that emodin attenuated the above-mentioned lung fibrosis and improve pulmonary functions by activating Sirt1 signaling to deacetylate R-Smad-induced ECM synthesis and deposition.

\section{References}

1. Iossifova Y, Bailey R, Wood J and Kreiss K: Concurrent silicosis and pulmonary mycosis at death. Emerg Infect Dis 16: 318-320, 2010. 
2. Hayes D Jr, Hayes KT, Hayes HC and Tobias JD: Long-term survival after lung transplantation in patients with silicosis and other occupational lung disease. Lung 193: 927-931, 2015.

3. Leung CC, Yu IT and Chen W: Silicosis. Lancet 379: 2008-2018, 2012.

4. Song J, Rong Y, Cui X and Chen W: Advances in research on the role of Gas 6/TAMin inflammation response and silicosis induced by silica dusts. Zhonghua Lao Dong Wei Sheng Zhi Ye Bing Za Zhi 32: 715-718, 2014 (In Chinese).

5. Gera K, Pilaniya V and Shah A: Silicosis: Progressive massive fibrosis with eggshell calcification. BMJ Case Rep 2014: bcr2014206376, 2014.

6. Weng ZP, Zhang JJ, Liu WW, Chen J, Liu YM, Yu W, Tang LJ, Chen JY, Fang M, Zhang C, et al: The experimental study of suppressing silicosis fibrosis. Zhonghua Lao Dong Wei Sheng Zhi Ye Bing Za Zhi 29: 740-745, 2011 (In Chinese).

7. Beamer CA, Migliaccio CT, Jessop F, Trapkus M, Yuan D and Holian A: Innate immune processes are sufficient for driving silicosis in mice. J Leukoc Biol 88: 547-557, 2010.

8. Davis GS, Pfeiffer LM, Leslie KE and Hemenway DR: Macrophage-lymphocyte cytokine interactions in silicosis. Chest 109 (3 Suppl): 49S-50S, 1996.

9. Allison SJ: Fibrosis: Regulation of fibrotic signalling by TGF- $\beta$ receptor tyrosine phosphorylation. Nat Rev Nephrol 10: 484, 2014.

10. Gao ZQ and Wang CH: Emodin and organ fibrosis. Zhongguo Zhong Xi Yi Jie He Za Zhi 25: 1030-1032, 2005 (In Chinese).

11. Chen XH, Sun RS, Hu JM, Mo ZY, Yang ZF, Jin GY, Guan WD and Zhong NS: Inhibitory effect of emodin on bleomycin-induced pulmonary fibrosis in mice. Clin Exp Pharmacol Physiol 36: 146-153, 2009.

12. Liu C: Inhibition of mechanical stress-induced hypertrophic scar inflammation by emodin. Mol Med Rep 11: 4087-4092, 2015.

13. Pooja $\mathrm{T}$ and Karunagaran D: Emodin suppresses Wnt signaling in human colorectal cancer cells SW480 and SW620. Eur J Pharmacol 742: 55-64, 2014.

14. Wang T, Zhong XG, Li YH, Jia X, Zhang SJ, Gao YS, Liu M and Wu RH: Protective effect of emodin against airway inflammation in the ovalbumin-induced mouse model. Chin J Integr Med 21: 431-437, 2015.

15. Huang XZ, Wen D, Zhang M, Xie Q, Ma L, Guan Y, Ren Y, Chen J and Hao CM: Sirtl activation ameliorates renal fibrosis by inhibiting the TGF- $/$ /Smad3 pathway. J Cell Biochem 115: 996-1005, 2014.

16. Zerr P, Palumbo-Zerr K, Huang J, Tomcik M, Sumova B, Distler O, Schett $G$ and Distler JH: Sirt1 regulates canonical TGF- $\beta$ signalling to control fibroblast activation and tissue fibrosis. Ann Rheum Dis $75: 226-233,2016$.
17. Wang T, Cui H, Ma N and Jiang Y: Nicotinamide-mediated inhibition of SIRT1 deacetylase is associated with the viability of cancer cells exposed to antitumor agents and apoptosis. Oncol Lett 6: 600-604, 2013.

18. Santos-Silva MA, Pires KM, Trajano ET, Martins V, Nesi RT, Benjamin CF, Caetano MS, Sternberg C, Machado MN, Zin WA, et al: Redox imbalance and pulmonary function in bleomycin-induced fibrosis in C57BL/6, DBA/2 and BALB/c mice. Toxicol Pathol 40: 731-741, 2012.

19. Subra JF, Renier G, Reboul P, Tollis F, Boivinet R, Schwartz P and Chevailler A: Lymphopenia in occupational pulmonary silicosis with or without autoimmune disease. Clin Exp Immunol 126: 540-544, 2001.

20. O'Connell M and Kennedy M: Progressive massive fibrosis secondary to pulmonary silicosis appearance on F-18 fluorodeoxyglucose PET/CT. Clin Nucl Med 29: 754-755, 2004.

21. Kitaguchi Y, Fujimoto K, Hanaoka M, Honda T, Hotta J and Hirayama J: Pulmonary function impairment in patients with combined pulmonary fibrosis and emphysema with and without airflow obstruction. Int J Chron Obstruct Pulmon Dis 9: 805-811, 2014.

22. Borthwick LA, Wynn TA and Fisher AJ: Cytokine mediated tissue fibrosis. Biochim Biophys Acta 1832: 1049-1060, 2013.

23. Tatler AL and Jenkins G: TGF- $\beta$ activation and lung fibrosis. Proc Am Thorac Soc 9: 130-136, 2012.

24. Cutroneo KR: TGF-beta-induced fibrosis and SMAD signaling: Oligo decoys as natural therapeutics for inhibition of tissue fibrosis and scarring. Wound Repair Regen 15 (Suppl 1): S54-S60, 2007.

25. Roberts AB, Tian F, Byfield SD, Stuelten C, Ooshima A, Saika $S$ and Flanders KC: $\operatorname{Smad} 3$ is key to TGF-beta-mediated epithelial-to-mesenchymal transition, fibrosis, tumor suppression and metastasis. Cytokine Growth Factor Rev 17: 19-27, 2006

26. Gauldie J, Bonniaud P, Sime P, Ask K and Kolb M: TGF-beta, Smad3 and the process of progressive fibrosis. Biochem Soc Trans 35: 661-664, 2007.

27. Yu JL, Li JH, Chengz RG, Ma YM, Wang XJ and Liu JC: Effect of matrine on transforming growth factor $\beta 1$ and hepatocyte growth factor in rat liver fibrosis model. Asian Pac J Trop Med 7: 390-393, 2014.

28. Chen N, Geng Q, Zheng J, He S, Huo X and Sun X: Suppression of the TGF- $\beta / \mathrm{Smad}$ signaling pathway and inhibition of hepatic stellate cell proliferation play a role in the hepatoprotective effects of curcumin against alcohol-induced hepatic fibrosis. Int J Mol Med 34: 1110-1116, 2014. 